\title{
Experimental and Simulation Analysis of Thermal Vertical Directional Solidification Grown Ni Doped Gasb
}

\author{
Sushanta K. Kamilla, Farida A. Ali
}

\begin{abstract}
Due to high hole mobility, p-GaSb is an attractive III-V semiconducting material for high performance p-channel metal-oxide semiconductor field effect transistor (p-MOSFET). For that growth of undoped and Ni doped GaSb bulk crystal by thermal vertical directional solidification technique has been reported in this paper. $X$-ray diffraction $(X R D)$ analysis confirms that the both grown compounds are polycrystalline in nature which have been supported by scanning electron microscope (SEM) image. The carrier charge density and mobility were measured by Hall Effect measurement in the temperature range $78 \mathrm{~K}$ and $300 \mathrm{~K}$. From the sign of Hall co-efficient the both grown materials were confirmed p-type. The Current-Voltage (I-V) characteristic was studied for both the sample i.e. experimentally grown and also simulated using TCAD at $78 \mathrm{~K}$ and $300 \mathrm{~K}$. The temperature dependence of the hole mobility were also investigated by TCAD tool using the models Auger recombination, Shockley-Read-Hall (SRH) and Band-gap narrowing $(B G N)$. Comparison of experimental and simulated temperature dependencies of mobility shows good agreement, while their differences at some points suggests the contribution of compensating impurities.
\end{abstract}

Keywords: p-GaSb, Thermal vertical directional solidification technique (TVDS), Hall Effect, I-V study, TCAD, SILVACO.

\section{INTRODUCTION}

Gallium Antimonide $(\mathrm{GaSb})$ is an attractive candidate for future high performance III-V p-channel metal-oxide semiconductor field effect transistor (p-MOSFET) because of its high hole mobility $(\sim 1000 \mathrm{~cm} 2 / \mathrm{V}$. Sec) which is higher than those of most of the other III-V semiconductors. Apart from that it is used as high speed electronic devices 5]. Also the trap neutral level (E0) of $\mathrm{GaSb}$ is located $0.1 \mathrm{eV}$ above the valence band, which is helpful in making Ohmic contacts to p-type GaSb for the p-MOSFET applications. These properties are advantageous for choosing $\mathrm{GaSb}$ and GaSb-based alloys also as promising channel materials in $\mathrm{p}$ MOSFETs [6]. Thus, development of p-MOSFETs using new material sets is an important goal to provide further scaling of CMOS circuits. Intense research activity also has been going on transition metals doped GaSb for spintronic device applications i.e. combination of both magnetic as well as semiconducting properties [7-11]. So far there are limited numbers of literatures present on the study of $\mathrm{Ni}$ in $\mathrm{GaSb}$ [12]. For both spintronic and high speed device applications $\mathrm{Ni}$ is essential due to its strong ferromagnetic property. Growth of bulk Ga1-xNixSb with different $\mathrm{Ni}$

Sushanta K. Kamilla, Semiconductors Research Lab, Institute of Technical Education and Research, Sikha 'O' Anusandhan (Deemed to be University), Bhubaneswar, Odisha, India.(Email: sushantakamilla@soa.ac.in)

Farida A. Ali, Semiconductors Research Lab, Institute of Technical Education and Research, Sikha ' $O$ ' Anusandhan (Deemed to be University), Bhubaneswar, Odisha, India. due to its narrow and direct band gap of $0.7 \mathrm{eV}$ at $300 \mathrm{~K}$ [1-

Revised Manuscript Received on September 14, 2019.

concentrations by vertical Bridgman method and then its detail electrical and magnetic characterization at $78 \mathrm{~K}$ and $300 \mathrm{~K}$ has been reported in one of our papers earlier [13].

In this paper we report on large grain polycrystals of $\mathrm{Ni}$ doped $\mathrm{GaSb}$ was grown by thermal vertical directional solidification (TVDS) technique designed and developed in our laboratory. The structural characterization was done by $\mathrm{X}$-ray diffraction (XRD) analysis and surface morphology was analyzed by using scanning electron microscope (SEM). The resistivity of the sample was determined by van der Pauw technique. Hall measurement was performed to determine the type of charge carriers, the carrier concentration and mobility. Undoped GaSb crystal was also grown using the same growth technique and characterized for comparison, with $\mathrm{Ni}$ doped GaSb. Later on simulation was carried out to study the ideal behavior of the undoped and $\mathrm{Ni}$ doped $\mathrm{GaSb}$ material so that comparison can be made with that of our grown materials. SILVACO-TCAD the simulation software which is the state-of-the-art available for academic purpose has been used for this study. This software tool is comprised of several modules designed to generate the geometry, doping, mesh, and perform the simulation by finite element method [14].

\section{GROWTH AND SIMULATION}

Using the thermal vertical directional solidification (TVDS) method two samples were grown. One is undoped $\mathrm{GaSb}$ for which the starting elements chosen are Gallium $(5 \mathrm{~N})$ and Antimony $(5 \mathrm{~N})$. For the second sample the starting elements chosen were Gallium $(5 \mathrm{~N})$, Antimony $(5 \mathrm{~N})$ and Nickel (4N) to grow Ni-doped GaSb. The starting elements for both the samples were taken from Johnson Matthey Co, USA. They were degreased by dipping in warm trichloroethylene, acetone and methanol and to remove the surface oxides. The starting elements were leached in dilute HCI solution with the help of ultrasonic vibrator (JULABO, Germany) followed by repeatedly washed with de-ionized water $(10 \mathrm{M} \Omega)$ and then vacuum dried in an oven. They were then put into capsules high purity quartz ampoules and vacuum-sealed to a pressure of $8 \times 10-4 \mathrm{~Pa}$ using a Hind high vacuum system model VS-65 (India) separately.

The quartz capsules were then placed inside a horizontal resistively heated furnace and were kept at $850^{\circ} \mathrm{C}$ for 3 hours with constant stirring with the help of rotational motor and nicrome net for a homogeneous mixture for the synthesis. Both the synthesized charges were then taken out from the capsules and again put inside two new conical tip quartz ampoules with inner diameter of $0.8 \mathrm{~cm}$ separately, 
followed by vacuum sealing to a pressure of 6x10-6 Torr. For growth the ampoule with synthesized material was then placed inside the TVDS technique furnace. Both undoped and $\mathrm{Ni}$ doped $\mathrm{GaSb}$ crystals were grown at the same growth conditions. Then the ingots were taken out by breaking the ampoules without any sticking problem to the wall for further processing and characterizations. Ingots of nearly $4 \mathrm{~cm}$ length and $0.8 \mathrm{~cm}$ diameter were obtained as shown in Figure 1.

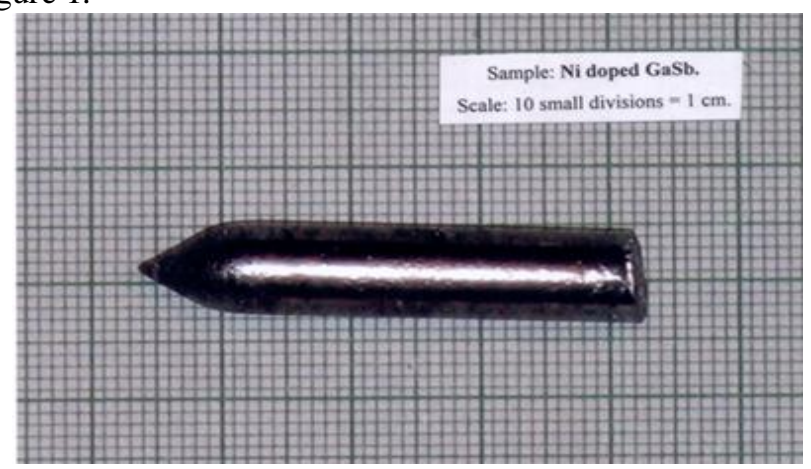

Figure 1: Photograph of grown Ni doped GaSb ingot.

Finally the wafers were cut perpendicular to the axis of the ingots with a slow speed diamond saw cutter. The saw damages were removed by mechanical polishing followed by chemical polishing to obtain a clean and smooth surface. The polished wafers were then chemically cleaned by dilute $\mathrm{HCl}$ to remove the instable native oxide layers and then washed to remove the traces of the chemicals and were finally vacuum dried in oven. The appropriate etchant is prepared by mixing proper proportional combination of $\mathrm{HNO} 3, \mathrm{HF}$ and $\mathrm{H} 2 \mathrm{O}$ to reveal the grains and grain boundaries that can be observed by using scanning electron microscope (SEM). Formation of the grown material was verified by X-ray diffraction (XRD) analysis. The XRD pattern of the material was taken by Rigaku miniflex X-Ray Diffraction Analyzer system by using a CuK $\alpha 1=1.5405 \AA$ radiation.

Resistivity and Hall Effect measurements with van der Pauw configurations were conducted for all samples using the Hall Instrument LAKESHORE 7504. The experiment was performed in the temperature range $78 \mathrm{~K}-300 \mathrm{~K}$. The electrical properties were also obtained from the simulation analysis of Ni doped GaSb by SILVACO-TCAD software tool. The simulation results obtained were then used for making comparisons with that of the experimental data. For this reason the size of simulation structure was taken of exact dimension (i.e. $5 \mathrm{~mm} \times 5 \mathrm{~mm}$ ) that corresponds to the experimental sample under test. Similarly, various material parameters at room temperature like doping type, doping level, carrier concentration etc. have been taken into consideration from experimental data. As the carrier concentration of the sample is about $1017 \mathrm{~cm}-3$ and the electrical characterization has been studied under the influence of temperature variation therefore the choice of Auger recombination for simulation study was essentially vital. Similarly the Shockley-Read-Hall (SRH) or trap related recombination is also dependent on material quality. Therefore, to study the ideal behavior of Ni doped GaSb, inclusion of these models to the simulated structure was necessary. Apart from these, model for band-gap narrowing
(BGN model) is also essential to include since the band-gap variation is dependent on intrinsic concentration, which in turn depends on temperature $[14,16]$. Thus, these three models Auger recombination, Shockley-Read-Hall (SRH) and Band-gap narrowing (BGN) play an important role in this simulation for the electrical characterizations of the $\mathrm{Ni}$ doped GaSb structured sample. Finally to characterize the I$\mathrm{V}$ analysis, a DC potential was applied and current at different temperatures ranging from $78 \mathrm{~K}$ to $300 \mathrm{~K}$ were calculated. Later the variation of hole mobility was found for the same range of temperature.

\section{RESULTS AND DISCUSSIONS}

\subsection{Structural and Morphological analysis}

The formation of $\mathrm{Ni}$ doped $\mathrm{GaSb}$ compound was confirmed by X-ray powder diffraction (XRD) technique. The typical XRD pattern of $\mathrm{Ni}$ doped $\mathrm{GaSb}$ is shown in Figure 2. The compounds were observed to be polycrystalline and zincblend structure. The possible reasons for the polycrystallinity of the grown ingot might be due to (i) shape of the tip of the ampoule not appropriately fabricated for single nucleation (ii) unavoidable minimal mechanical vibration at the time of growth (iii) thermal convection. The lattice parameter of the cubic crystals was calculated and was found $\mathrm{a}=6.092 \AA$ for $\mathrm{Ni}$ doped $\mathrm{GaSb}$ and $\mathrm{a}=6.097 \AA$ for undoped $\mathrm{GaSb}$ ingot. The reported value of lattice constant for undoped sample a is $6.095 \AA$ [JCPDS070215]. The decrease in a value may be due to addition of $\mathrm{Ni}$ in the crystal lattice indirectly indicates inclusions of $\mathrm{Ni}$ in the GaSb lattice.

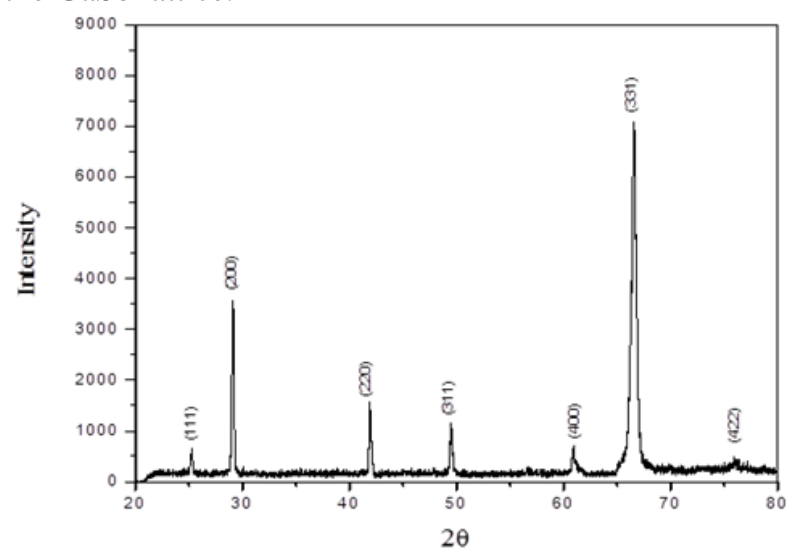

Figure 2. Typical $\mathrm{X}$ - Ray diffraction pattern using $\mathrm{Cu}$ $\mathrm{K} \alpha$ radiation for $\mathrm{Ni}$ doped $\mathrm{GaSb}$ sample.

The surface morphology of polished wafer of grown $\mathrm{Ni}$ doped GaSb sample is shown in Figure 3. The clear demarcations of grain and grain boundaries of the polycrystalline sample are shown. 


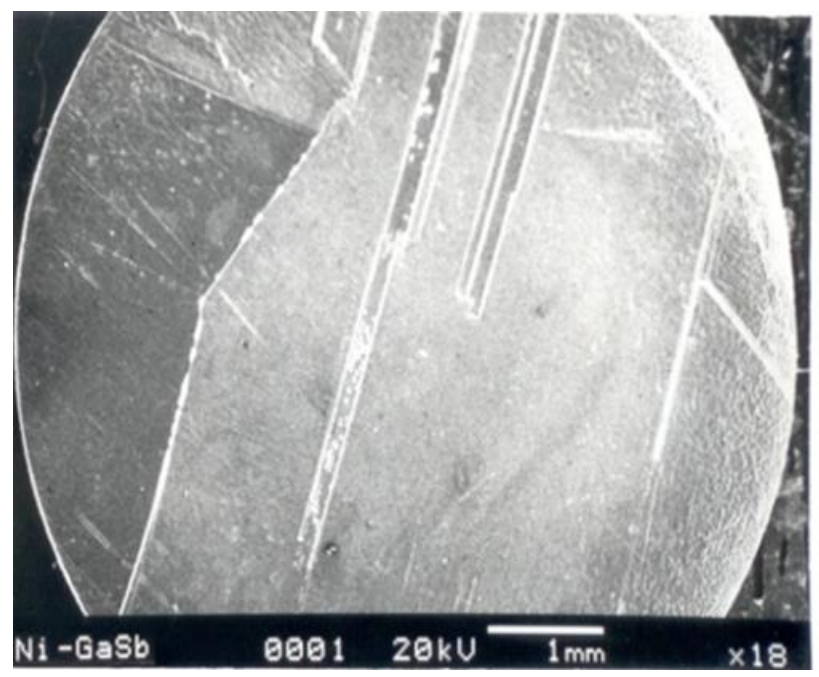

Figure 3.The photograph of polished wafer of grown Ni doped GaSb sample

The average grain size of the ingot is nearly $2.6 \mathrm{~cm}$. Both Roy and Basu [16] and Harsy et.al [18] have reported that the grain size strongly depends upon the ampoule diameter. The decrease in grain size with lower ampoule diameters was explained as due to the wall effect, whereas for large ampoule diameter non uniformity of heat conduction from the crystal to the melt interface results in decrease in grain size. The presence of dopant impurity $\mathrm{Ni}$ has also strong influence on the grain size of the ingot grown by vertical Bridgman method. Furthermore, the growth temperature may also be one of the reasons for the formation polycrystalline $\mathrm{Ni}$ doped $\mathrm{GaSb}$ sample i.e. the thermo mechanical stress occurring during the cooling condition. This suggests that observed morphology was probably due to the stress and the defects joined up to produce a higher number of grains and grain boundaries. Secondly, due to the incorporation of dopant atoms strain develops because of the difference in the atomic radius of the dopants and the host atoms.

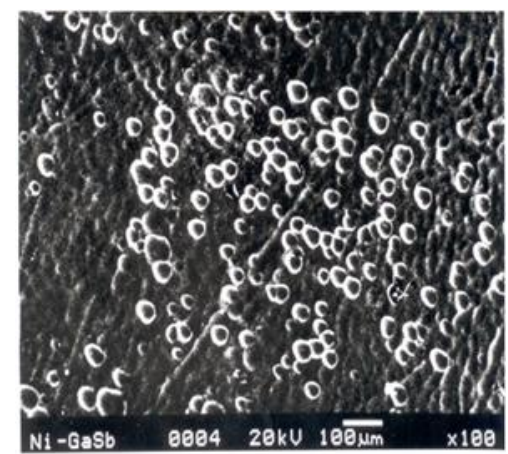

Figure 4.Cross sectional view of Ni doped GaSb sample showing the EPDs

The etched wafer of grown sample was analyzed by SEM and the average EPD was found to be $1.5 \times 109 / \mathrm{m} 2$. This suggests that probably due to stress and strain at the time of growth grains and grain boundaries were formed. The etch rate of an anisotropic etchant on zinc blende type materials are slowest and also the pits are triangular on [111] surface as observed in Figure 4.
3.2 Electrical Characterizations of both experimental \& simulated sample

The resistivity of the sample was determined by Van Der Pauw technique. The first step for preparing the sample was to make a proper ohmic contact by melting high purity Indium metal dots on the corners of the rectangular sample and it was verified. Hall measurement was performed to determine the type of charge carriers, carrier concentration and mobility using the same configuration. The sample was found to be p-type as confirmed by the sign of Hall coefficient and Hall co-efficient also decreases from 111.6 $\mathrm{cm} 3 / \mathrm{C}$ to $25.3 \mathrm{~cm} 3 / \mathrm{C}$ with the rise of temperature from $78 \mathrm{~K}$ to $300 \mathrm{~K}$.

The study of I-V characteristics of the experimental sample at $78 \mathrm{~K}$ and $300 \mathrm{~K}$ is found to be linear and passes through origin as shown in Figure 5(a). The simulated I-V characteristic was also studied for a similar rectangular sized $\mathrm{Ni}$ doped GaSb sample shown in Figure 5(b) at both $78 \mathrm{~K}$ and $300 \mathrm{~K}$. The resistance of the simulated sample was found out from the slopes of the two curves. The result shows that at $78 \mathrm{~K}$ i.e. at low temperature the charge carrier holes are not free for conduction thus the sample showing lower conductivity as compared to the experimental sample. For a higher temperature the hole concentration increases rapidly because the charge carriers are thermally excited into the conduction band and as a result the conductivity increases rapidly. At $78 \mathrm{~K}$ and $300 \mathrm{~K}$ the electrical parameters for both experimental and simulated samples of $\mathrm{Ni}$ doped $\mathrm{GaSb}$ have been summarized in Table 1 . The results indicate that resistivity, mobility, while the hole concentration increases for both the samples.

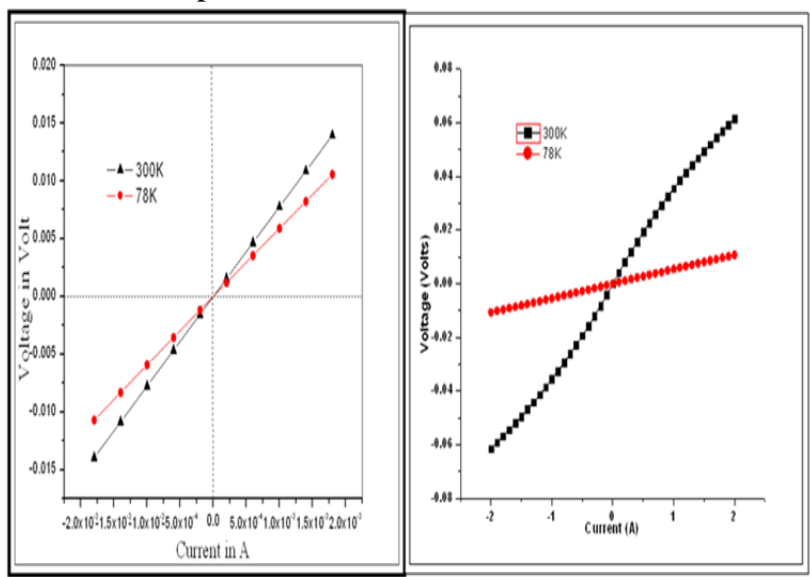

Figure 5. Current (I) - voltage (V) characteristics for the Indium ohmic contact to Ni doped GaSb at $300 \mathrm{~K}$ and 78K (a) Experimental (b) Simulation results 
Table 1. Electrical parameters at $300 \mathrm{~K}$ and $78 \mathrm{~K}$

\begin{tabular}{|c|c|c|c|}
\hline Samples & \multicolumn{2}{|c|}{ Resistivity (Ocm) } & \multicolumn{2}{r|}{ hole conce } \\
(cm
\end{tabular}

Figure 6 (a) shows a variation of mobility in the temperature range $78 \mathrm{~K}-300 \mathrm{~K}$ for the experimental sample. The mobility of the hole does not show any significant change between $78 \mathrm{~K}$ and $240 \mathrm{~K}$ and after that the mobility decreases with temperature. The reason for this behavior can be attributed to the fact that as temperature increases, phonon scattering starts increasing and it reduces the drift of the carriers and thus the mobility. At $300 \mathrm{~K}$ the hole mobility of $\mathrm{Ni}$ doped $\mathrm{GaSb}$ is $434 \mathrm{~cm} 2 / \mathrm{V}$.sec whereas the hole mobility for the simulated $\mathrm{Ni}$ doped sample is found to be $395 \mathrm{~cm} 2 / \mathrm{V} . \mathrm{sec}$. The simulated result of hole mobility of undoped bulk GaSb was reported as $458 \mathrm{~cm} 2 / \mathrm{V} . \mathrm{sec}$ earlier. This variation in simulated undoped and dopoed $\mathrm{GaSb}$ indicates that the hole mobility decreases due to the impurity scattering effect of Ni concentration. Here we have observed the highest mobility of $1096 \mathrm{~cm} 2 / \mathrm{V} . \mathrm{sec}$ at $93 \mathrm{~K}$ for the experimental sample of $\mathrm{Ni}$ doped GaSb. Our experimental observation agrees with the reported results by Stollwerck. [15].

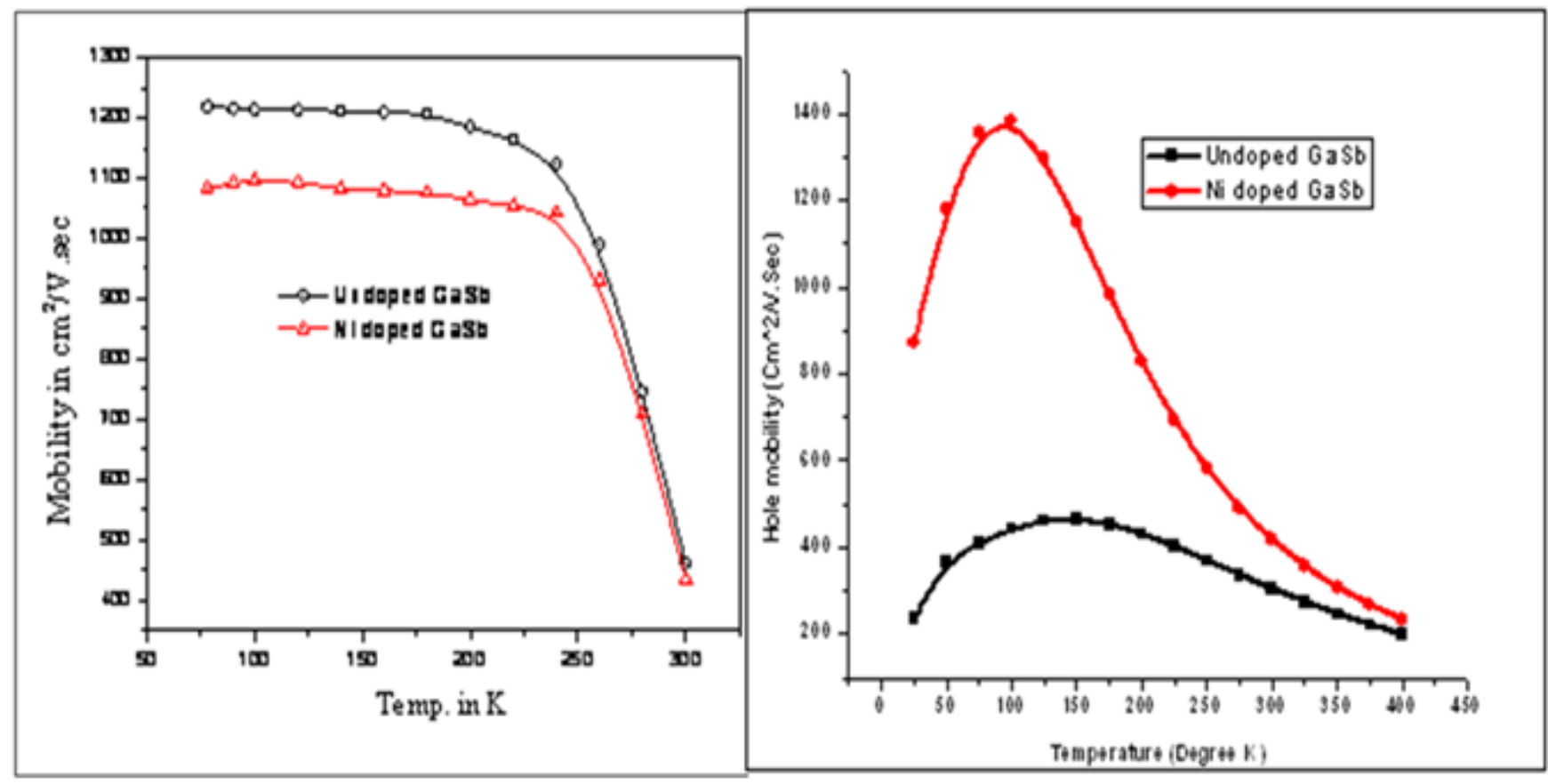

(b)

Figure 6. Variation of mobility with temperature of Undoped and Ni doped GaSb at $300 \mathrm{~K}$ and 78K (a) Experimental (b) Simulation results

Whereas Figure 6 (b) shows a variation of mobility in the temperature range $78 \mathrm{~K}-300 \mathrm{~K}$ for the simulated one. In general the undoped $\mathrm{GaSb}$ samples show higher peak mobility than the doped GaSb samples due to the compensation mechanism present [18]. But our simulated $\mathrm{Ni}$ doped sample, though it shows the similar trend but shows higher mobility than that of undoped sample, this is because we have not included any model to take care the compensation mechanism. Therefore, the sample shows a mobility of $1371 \mathrm{~cm} 2 / \mathrm{V} . \mathrm{sec}$ at $93 \mathrm{~K}$ which is much higher than our experimental mobility.

\section{CONCLUSION}

From the presented study the vertical Bribgeman growth technique of both undoped and $\mathrm{Ni}$ doped $\mathrm{GaSb}$ have been analyze. The presence of dopant impurity $\mathrm{Ni}$ has strong influence on the polycrystallinity nature and grain size of the ingot grown by vertical Bridgman method. This has been confirmed from the XRD study and the photograph of etched wafer from the grown ingot respectively. Later on both experimentally and by simulation electrical characterization was done considering Nickel as a dopant in bulk GaSb for $78 \mathrm{~K}$ and $300 \mathrm{~K}$. Nickel behaves as an acceptor impurity and also reduces the hole mobility of $\mathrm{GaSb}$. Experimental and simulation results were compared for the temperature dependencies of mobility. The results show good agreement, while the differences may be due to the scattering and compensation mechanisms.

\section{ACKNOWLEDGEMENT}

The corresponding authors, Dr.S.K.Kamilla is thankful to Indian Institute of Technology (IIT), Kharagpur, India for doing laboratory and other facilities.

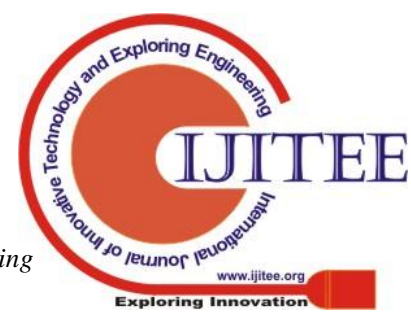




\section{REFERENCES}

1. Dutta P. S. et. al. 1997 Journal of Applied Physics 819 5821

2. Ancona M.G. et. al. 2010 Solid-State Electronics 541349

3. Zhao Lian-Feng et. al. 2015 Chinese Physics. B 241 018501

4. Yuan Ze et. al. 2013 IEEE Electron Device Letters 3411 1367

5. Ali F. A. et. al. 2011 IEEE Students' Technology Symposium (TechSym) 211

6. Zota B. Cezar et. al. 2012 Applied Physics Express 5 071201

7. Sestakova V. et. al. 1995 Journal of Crystal Growth 146 87

8. Ganesan K et. al. 2011 Journal of Applied Physics 109 033903

9. Matsukura F. et. al. 2000 Journal of Applied Physics 879 6642

10. Hidalgo P. et. al. 1999 Physics Review B 601510613

11. Karar N. et. al. 1999 Materials Science and Engg. B 56 21

12. Kamilla S.K. et. al. 2014 Advanced Science Letters, 20 3-4 784

13. Kamilla S.K. et. al. 2011 Solid State Sciences 13232

14. Atlas user manual, Chapter 3 (2006)

15. Stollwerck G. 2000 IEEE Transactions on Electron Devices 472448

16. Roy U.N et. al. 1988 Materials Letters 6238

17. Harsy M. et. al. 1981 Journal of Crystal Growth 53234. 